\title{
Collimated Multi-MeV Ion Beams from High-Intensity Laser Interactions with Underdense Plasma
}

\author{
L. Willingale, ${ }^{1}$ S. P. D. Mangles, ${ }^{1}$ P. M. Nilson, ${ }^{1}$ R. J. Clarke, ${ }^{2}$ A. E. Dangor, ${ }^{1}$ M. C. Kaluza, ${ }^{1}$ S. Karsch, ${ }^{3}$ K. L. Lancaster, ${ }^{2}$ \\ W. B. Mori, ${ }^{4}$ Z. Najmudin, ${ }^{1}$ J. Schreiber, ${ }^{3,5}$ A. G. R. Thomas, ${ }^{1}$ M. S. Wei, ${ }^{1, *}$ and K. Krushelnick ${ }^{1}$ \\ ${ }^{1}$ Blackett Laboratory, Imperial College London, London SW7 2BZ, United Kingdom \\ ${ }^{2}$ Central Laser Facility, Rutherford-Appleton Laboratory, Chilton, Oxon, OX11 OQX, United Kingdom \\ ${ }^{3}$ Max-Planck Institut für Quantenoptik, Hans-Kopfermann-Straße 1, D-85748 Garching, Germany \\ ${ }^{4}$ Department of Physics and Astronomy \& Department of Electrical Engineering, UCLA, Los Angeles, California 90095, USA \\ ${ }^{5}$ Ludwig-Maximilians-Universität München, Am Coulombwall 1, D-85748 Garching, Germany
}

(Received 14 December 2005; published 22 June 2006)

\begin{abstract}
A beam of multi-MeV helium ions has been observed from the interaction of a short-pulse highintensity laser pulse with underdense helium plasma. The ion beam was found to have a maximum energy for $\mathrm{He}^{2+}$ of $\left(40_{-8}^{+3}\right) \mathrm{MeV}$ and was directional along the laser propagation path, with the highest energy ions being collimated to a cone of less than $10^{\circ}$. $2 \mathrm{D}$ particle-in-cell simulations show that the ions are accelerated by a sheath electric field that is produced at the back of the gas target. This electric field is generated by transfer of laser energy to a hot electron beam, which exits the target generating large spacecharge fields normal to its boundary.
\end{abstract}

DOI: 10.1103/PhysRevLett.96.245002

PACS numbers: 52.38.Kd, 52.38.Hb, 52.65.Rr

With the development of short-pulse high-intensity lasers it has become possible to generate energetic particles such as relativistic electrons [1], $\gamma$ rays [2], and $\mathrm{MeV}$ ions [3-5] from laser-plasma interactions. The acceleration of ions has received particular attention due to the high quality of the energetic beams produced. The ion beams produced are charge neutralized and are found to have high energy, large total charge, small emittance [6], and small virtual source size [7]. This makes them particularly valuable for many proposed applications such as proton radiography [7], isochoric heating [8], medical isotope preparation [9], cancer therapy [10], and even as possible injectors for higher energy accelerators [11].

Laser-plasma interactions can be broadly categorized into either underdense or overdense interactions depending on whether or not the laser can propagate through the plasma. Most recent investigations of ion acceleration from a laser-plasma interaction have concentrated on overdense plasmas, and, in particular, on the beam of ions emitted from the rear surface of laser irradiated solid targets. The ions are accelerated by the large quasistationary space-charge fields set up at both front and rear surfaces of the target or by electrostatic shocks propagating through the target. The space-charge separation at the front surface is due to the ponderomotive potential of the laser pulse $[12,13]$. At the rear surface, the accelerating field is set up by the expulsion into vacuum of the beam of hot electrons which is generated at the front surface and transported through the target [14]. The balance between the front and rear acceleration fields is determined by the laser conditions, in particular, the prepulse level, laser intensity, and target geometry [15]. The ion beam primarily consists of protons which are found as water and organic contami- nants on most solid targets. Thus to accelerate higher $Z$ ions efficiently, significant effort must be taken to prepare and clean the targets before irradiation $[13,16]$.

Multi-MeV energy ions have also been observed from underdense plasma targets [17]. In this case the ions were found to be emitted transverse to the direction of laser beam propagation. A Coulomb explosion [18] accelerates these ions through the large space-charge field generated by the ponderomotive expulsion of electrons from the central channel by the high-intensity laser. Recently, it has been observed that the maximum energy of the transverse ions is increased by collisionless shock acceleration [19]. This underdense ion acceleration mechanism allows ions from gas of any required $Z$ to be efficiently accelerated. However, the lack of collimation of the ions limits the use of this mechanism for most applications.

In this Letter we present the first observation of a collimated beam of $\mathrm{MeV}$ ions generated in the forward direction from underdense laser-plasma interactions. The balance between transverse and longitudinal acceleration is found to be primarily dependent on plasma density. At the highest density investigated, the forward going ion beam was found to have a maximum energy much higher than the transverse ion beam from the same interaction. Twodimensional particle-in-cell (PIC) simulations were used to investigate the effect of the plasma density on the acceleration of the ions in the forward direction. It is found that the longitudinally accelerated ions are generated by a sheath acceleration mechanism, similar to those generated in solid target interactions. The laser energy is efficiently absorbed mainly by direct laser acceleration of the plasma electrons to energies up to hundreds of $\mathrm{MeV}$ [20]. As the fast electrons leave the plasma, they generate a large 
electric field, which accelerated ions to energies of many $\mathrm{MeV}$. The simulations show a collimated beam of ions with a $7^{\circ}$ half-angle divergence.

The experiment was performed using the VULCAN Petawatt laser [21]. The laser pulse had a duration of $\tau_{l} \simeq$ $1.0 \mathrm{ps}$ full width half maximum (FWHM) with energy of up to $340 \mathrm{~J}$ on target and a central wavelength $\lambda_{0}=$ $1.054 \mu \mathrm{m}$. An $f / 3$ off-axis parabolic mirror focused the laser onto the edge of a gas jet with a $2 \mathrm{~mm}$ diameter supersonic nozzle. The laser intensity profile at vacuum focus was characterized by a FWHM of $w_{0} \simeq 7 \mu \mathrm{m}$. The peak vacuum intensity of the laser was therefore $I_{0} \simeq$ $5.5 \times 10^{20} \mathrm{~W} \mathrm{~cm}^{-2}$, which corresponds to a peak normalized vector potential, $a_{0} \simeq 21$. The backing pressure to the gas-jet nozzle was varied so that the electron density of the fully ionized plasma could be set between $(0.7-4.0) \times$ $10^{19} \mathrm{~cm}^{-3}$. Supersonic nozzles were used to ensure a uniform density profile over a given distance (depending on the diameter of the nozzle), and a reasonably sharp density ramp both at the front and back of the gas jet $(<250 \mu \mathrm{m})$. The density profile of the gas jet was determined prior to the high-intensity shots by interferometry. The frequency separation of the forward Raman scattered laser spectra $\left[\Delta \omega=\omega_{p e}=\left(n_{e} e^{2} / m_{e} \epsilon_{0}\right)^{1 / 2}\right]$ confirmed the electron density $n_{e}$ of the interaction.

Energy spectra of accelerated ions were taken at four different angles to measure the angular emission of the ions. Thomson parabola ion spectrometers were placed at $45^{\circ}$ and $90^{\circ}$ to the direction of the laser propagation. In addition, there were two magnetic spectrometers, one placed at $10^{\circ}$ and one directly on the axis of propagation of the laser $\left(0^{\circ}\right)$. Though primarily used to measure the spectrum of energetic electrons emitted in the interaction, the open geometry of the magnetic spectrometers also allowed the measurement of ions and other positively charged particles. The ions are deflected in the opposite direction from electrons by the magnetic field between the spectrometer's circular pole pieces. The deflection of the ions depends simply on their charge-to-mass ratio and their momentum, thus allowing their energy to be determined. The energy resolution of the ion spectra was determined by the width of the entrance slit to the spectrometer, which was $2 \mathrm{~mm}$ wide. The nuclear track detector CR39, which is insensitive to positrons, was used to detect the ions. CR39 responds to ions with energy greater than $\sim 100 \mathrm{keV} /$ nucleon. The tracks caused by the passage of energetic ions can be revealed by etching the CR39 with $\mathrm{NaOH}$ solution.

For a helium gas target, there are two possible ion species, $\mathrm{He}^{1+}$ and $\mathrm{He}^{2+}$. In the Thomson parabola spectrometers, these different charge-to-mass ratio species are separated spatially by an additional electric field. In the magnetic spectrometers, the different species are not separated; therefore, it was necessary to distinguish between the species by the pit size for all ions deflected by a similar amount. A schematic of the experimental setup and the onaxis charged-particle spectrometer, measuring both the electrons and ions, is shown in Fig. 1. At a particular point on the detector, both ion species may be present but the $\mathrm{He}^{2+}$ will have more energy. The higher the energy of an ion, the further into the CR39 it will travel before it is stopped and therefore the damage will be deeper into the material. When the CR39 is etched, pits appear where the ions are stopped. The smaller pits on the surface are the result of deeper damage. Hence at a particular point, using the electron spectrometers, the smaller pits will be from $\mathrm{He}^{2+}$ ions and the larger ones from $\mathrm{He}^{1+}$ ions, allowing the species to be differentiated. Electron spectra were also measured simultaneously on each shot by using image plate detectors as has previously been reported [20].

For plasma densities below $2 \times 10^{19} \mathrm{~cm}^{-3}$ energetic ions were found to be emitted primarily in the transverse direction consistent with previous measurements [17,19]. However, above this density a clear, reproducible signal of ions was observed in the $0^{\circ}$ and $10^{\circ}$ spectrometers. For example, we present helium ion spectra from a shot for which a pulse of $340 \mathrm{~J}\left(I_{0} \approx 6 \times 10^{20} \mathrm{~W} \mathrm{~cm}^{-2}\right)$ was incident on a helium plasma with an electron density of $n_{e}=$ $4 \times 10^{19} \mathrm{~cm}^{-3}$. The electron spectrum for this shot has a maximum energy of $\sim 65 \mathrm{MeV}$, with a characteristic temperature of $T_{e}=7.4 \mathrm{MeV}$. The helium spectra at various angles are shown in Fig. 2. In the transverse direction $\mathrm{He}^{2+}$ had a maximum energy of $(7.8 \pm 0.6) \mathrm{MeV}$ and $\mathrm{He}^{1+}$ had a maximum energy of (3.4 \pm 0.4$) \mathrm{MeV}$. In the longitudinal direction $\mathrm{He}^{2+}$ had a maximum energy of $\left(40_{-8}^{+3}\right) \mathrm{MeV}$ and $\mathrm{He}^{1+}$ had a maximum energy of $(10 \pm 3) \mathrm{MeV}$. There were no pits observed on the back surface of the CR39 which means that there were no helium ions with $43 \mathrm{MeV}$, which gives an upper limit to the energy. Noticeably, the maximum energy and number at $45^{\circ}$ is less than both the transverse and longitudinal directions. Also, the maximum energy at $10^{\circ}$ is about an order of magnitude less in number than in the forward direction. Hence one can ascertain that ions in the range 5-40 $\mathrm{MeV}$ are collimated in a beam with divergence angle less than $10^{\circ}$ in the forward direction.

2D PIC simulations were performed with the code OSIRIS [22] to model this type of interaction. The laser was propagated through a stationary box to allow the ion

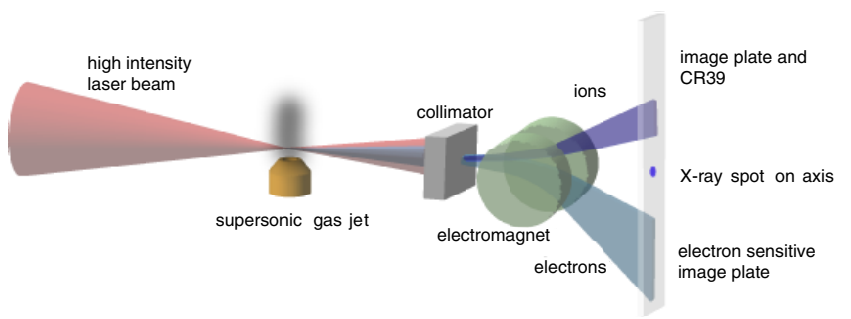

FIG. 1 (color online). Experimental setup showing the on-axis charged-particle spectrometer. 


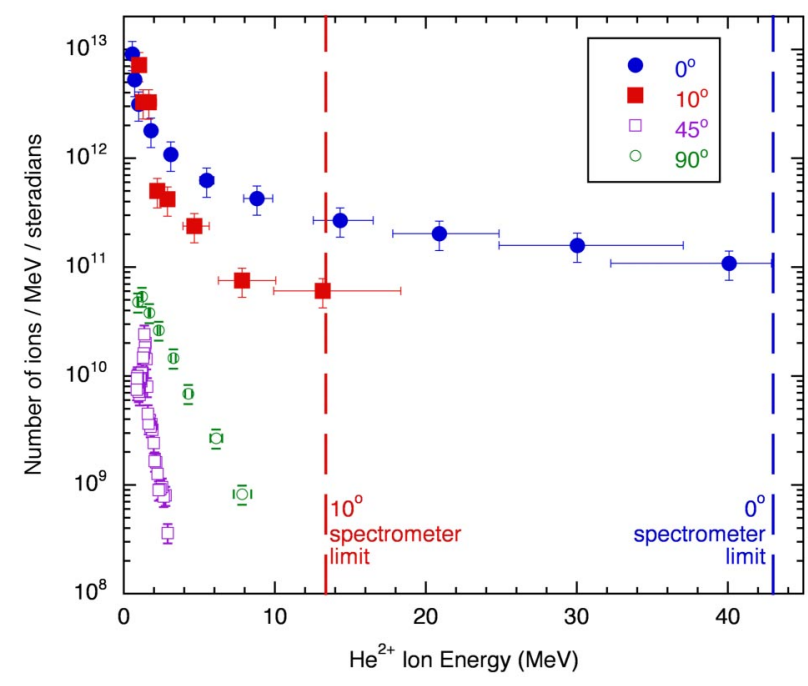

FIG. 2 (color online). Helium ion spectra at various angles.

dynamics to be observed to later times, even after the laser pulse had left the box. The box was $251 \times 251 \mu \mathrm{m}$ with a resolution of 20.9 cells $/ \lambda$ in the longitudinal $(x)$ direction and 12.6 cells $/ \lambda$ in the transverse direction $(y)$. The box was smaller than the experimental dimensions due to computational constraints. The ion acceleration was investigated with changing plasma electron density. Since ionization effects are not included in the code, a $\mathrm{He}^{2+}$ plasma was used to avoid complications due to balance of the different charge states. Electron densities of $0.1 n_{c}$, $0.05 n_{c}$, and $0.01 n_{c}$ were simulated, where $n_{c}$ is the critical density. The plasma profile had a $71 \mu \mathrm{m}$-long linear density ramp at the front, $25 \mu \mathrm{m}$ of plasma at maximum density and then $71 \mu \mathrm{m}$ density ramp at the back of the target. The vacuum then extended a further $84 \mu \mathrm{m}$ behind the plasma. The laser pulse was linearly polarized, with $\tau_{l}=500 \mathrm{fs}$ and focused to a spot of diameter $w_{0}=8 \mu \mathrm{m}$ at the top of the front density ramp to give a peak normalized vector potential $a_{0} \sim 13$.

Figure 3 shows the electron and ion densities and the longitudinal electric field at $1.0 \mathrm{ps}$ into the simulation, for a

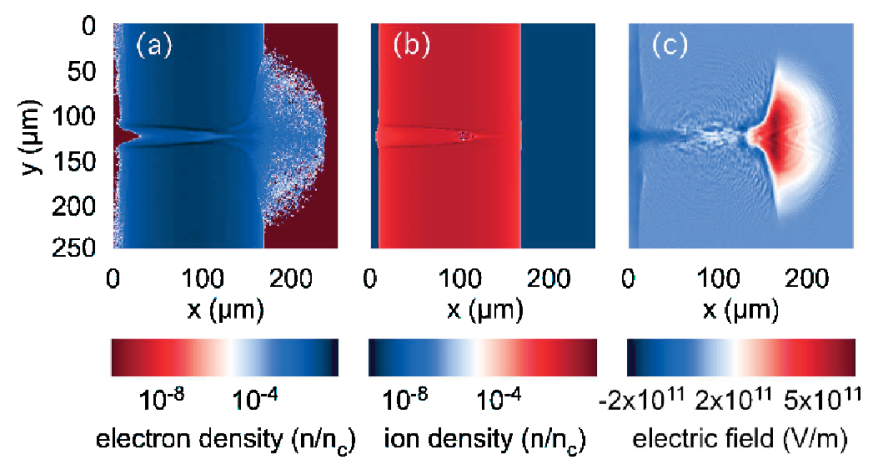

FIG. 3 (color). At a time 1.0 ps after the start of a simulation for $n_{e}=0.05 n_{c}$ (a) electron density, (b) $\mathrm{He}^{2+}$ ion density, and (c) longitudinal electric field. run with $n_{e}=0.05 n_{c}$. The electrons have been accelerated by a combination of plasma fields and direct laser acceleration. It is noted that PIC simulations provide a reasonable model of the transport of the hot electrons in underdense targets due to the relatively negligible effect of collisions. The high-charge high-energy electron beam diverges and finally moves out into the vacuum. Since the ions have not yet responded, a large longitudinal electric field $E_{x}$ is formed at the plasma-vacuum interface. The peak electric field $(\sim 0.7 \mathrm{TV} / \mathrm{m})$ occurs at a time close to that shown in Fig. 3. As the ions at the back of the target begin to move, in response to this field, $E_{x}$ is reduced in value. Nevertheless, the ions continue to be accelerated by the expanding sheath front.

Figure 4 shows the ion $p_{x}$ against $p_{y}$ momentum at times of $0.5 \mathrm{ps}, 1.0 \mathrm{ps}, 1.5 \mathrm{ps}, 2.5 \mathrm{ps}$, and $3.5 \mathrm{ps}$. Before the hot electron beam exits the plasma, acceleration is only seen in the transverse direction, $p_{y}$. This transverse acceleration is due to the "Coulomb explosion." Once the hot electrons leave the target and the back surface sheath field is set up, a beam of ions is accelerated in the longitudinal direction. The $\mathrm{He}^{2+}$ ion beam has a maximum energy of about $45 \mathrm{MeV}$ and a divergence of $7^{\circ}$ half-angle at $25 \mathrm{MeV}$ at the end of the simulation. It is also noted that in the simulations, the exit of the return current from the front of the target ejects ions from the front surface as well. However, with the thicker targets used, this feature has yet to be observed experimentally.

The simulated $\mathrm{He}^{2+}$ ion spectra, in both the longitudinal and transverse directions, for different density plasmas, are shown in Fig. 5. The density scan reveals the same dependence observed experimentally. At the lowest density $\left(0.01 n_{c}\right)$, the acceleration is almost purely in the transverse direction. However, at $0.05 n_{c}$ the longitudinal acceleration is already more effective than the radial acceleration. At the highest density of $0.1 n_{c}$, the radial ion acceleration is

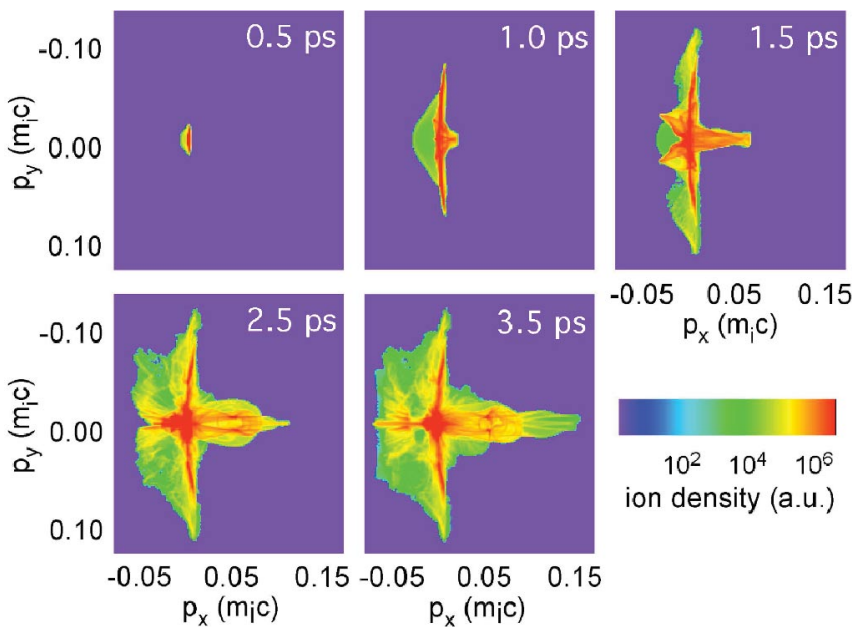

FIG. 4 (color). $\quad \mathrm{He}^{2+}$ ion $p_{x}$ against $p_{y}$ plots, at times of $0.5 \mathrm{ps}$, $1.0 \mathrm{ps}, 1.5 \mathrm{ps}, 2.5 \mathrm{ps}$, and $3.5 \mathrm{ps}$ for $n_{e}=0.05 n_{c}$. 


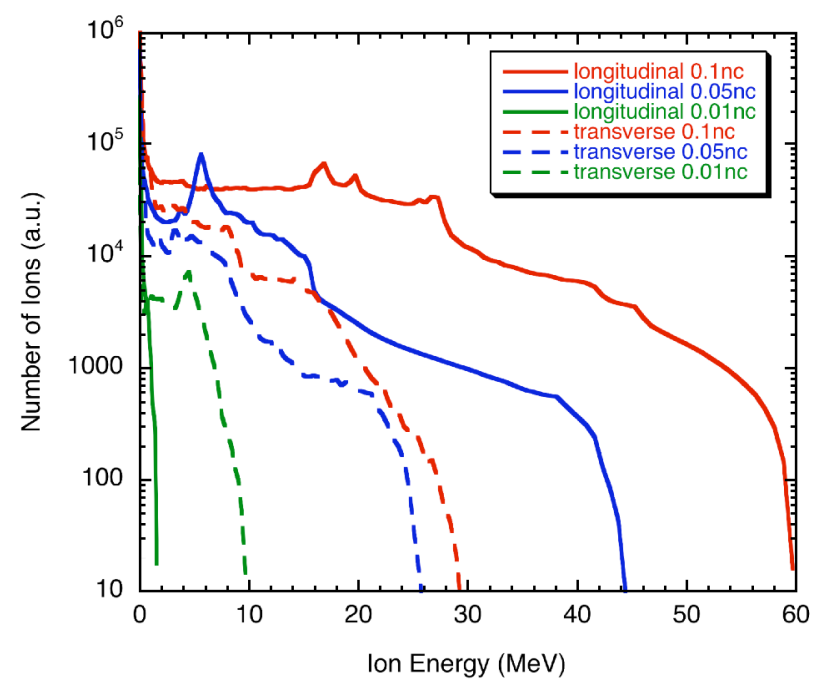

FIG. 5 (color online). Simulated $\mathrm{He}^{2+}$ ion spectra at $3.5 \mathrm{ps}$ for plasma densities of (a) $0.1 n_{c}$, (b) $0.05 n_{c}$, and (c) $0.01 n_{c}$.

again not as effective as the longitudinal ion acceleration in number or maximum energy. Indeed the amount of acceleration is limited in the simulations by the amount of vacuum behind the plasma (again due to computational constraints). With a longer acceleration length, the ions would attain even higher energies longitudinally.

The acceleration of ions by an electrostatic sheath can be modeled as the plasma expansion into a vacuum $[23,24]$. An expression for the maximum ion energy from this model is $\mathcal{E}_{\max } \simeq 2 Z k_{B} T_{e}\left[\ln \left(\tau+\sqrt{\tau^{2}+1}\right)\right]^{2}$, where $\tau=$ $\omega_{p i} t / \sqrt{2 e^{1}}, t$ is the time that the electrons are hot and $e^{1}$ denotes the numerical constant 2.71828.... At a plasma density of $4 \times 10^{19} \mathrm{~cm}^{-3}$ with a measured electron temperature of $T_{e}=7.4 \mathrm{MeV}$ and for a laser pulse length of 1 ps gives maximum ion energies greater than but of the order of those measured. It may be considered surprising that we observe ion energies of this order, considering that this model assumes a zero scale-length plasma on the rear surface. For solid density targets, increased rear-end density scale length has been shown to dramatically reduce the acceleration, since the maximum electric field is given by $E_{\max } \approx T_{e} /(e L)$, where $L$ is the sheath scale length $[14,15]$. In both our experiment and simulations, the scale length due to the initial gas density ramp is much larger than the Debye length $\lambda_{D}=\left(\epsilon_{0} k_{B} T_{e} / n_{e o} e^{2}\right)^{1 / 2}$, and may be expected to reduce the maximum electric field by greater than an order of magnitude. However, the simulations show that a larger fraction of the hot electrons can escape in the underdense case due to the high electron temperature. This is aided by the presence of the highintensity laser pulse in the sheath region, which opposes the space-charge force trying to prevent plasma electrons escaping to vacuum.

Because of the large radial extent of the sheath field in this interaction, a relatively long acceleration time overcomes the relatively low electric field strength, in underdense as opposed to overdense interactions. Furthermore, the high fast electron temperatures and almost uninhibited transport of the plasma electrons are also beneficial to the maximum final energies of the ions. Finally we note that underdense targets are relatively immune to detrimental prepulse effects and furthermore do not require complex target construction or extensive preshot cleaning as compared to solid targets. Hence gas targets may prove to be an interesting alternative for generating collimated beams of high-energy ions from laser-plasma interactions both for high $Z$ ions as well as protons. Since much higher electron temperatures are possible with gas targets it may be possible to accelerate ions to higher energies using this technique.

The authors acknowledge the staff of the Central Laser Facility (RAL) for technical assistance. We gratefully acknowledge the OSIRIS consortium (UCLA/IST/USC) for the use of OSIRIS.

*Present address: Center for Energy Research, University of California, San Diego, CA 92093-0417, USA.

[1] A. Modena et al., Nature (London) 377, 606 (1995).

[2] R. D. Edwards et al., Appl. Phys. Lett. 80, 2129 (2002).

[3] A. R. Bell et al., Phys. Rev. E 48, 2087 (1993).

[4] E. L. Clark et al., Phys. Rev. Lett. 84, 670 (2000).

[5] R. A. Snavely et al., Phys. Rev. Lett. 85, 2945 (2000).

[6] T. E. Cowan et al., Phys. Rev. Lett. 92, 204801 (2004).

[7] M. Borghesi et al., Phys. Rev. Lett. 92, 055003 (2004).

[8] P. K. Patel et al., Phys. Rev. Lett. 91, 125004 (2003).

[9] I. Spencer et al., Nucl. Instrum. Methods Phys. Res., Sect. B 183, 449 (2001).

[10] S. V. Bulanov et al., Phys. Lett. A 299, 240 (2002).

[11] K. Krushelnick et al., IEEE Trans. Plasma Sci. 28, 1184 (2000).

[12] A. Maksimchuk et al., Phys. Rev. Lett. 84, 4108 (2000).

[13] M. Zepf et al., Phys. Rev. Lett. 90, 064801 (2003).

[14] S. C. Wilks et al., Phys. Plasmas 8, 542 (2001).

[15] M. C. Kaluza et al., Phys. Rev. Lett. 93, 045003 (2004).

[16] M. Allen et al., Phys. Rev. Lett. 93, 265004 (2004).

[17] K. Krushelnick et al., Phys. Rev. Lett. 83, 737 (1999).

[18] G. S. Sarkisov et al., Phys. Rev. E 59, 7042 (1999).

[19] M. S. Wei et al., Phys. Rev. Lett. 93, 155003 (2004).

[20] S. P. D. Mangles et al., Phys. Rev. Lett. 94, 245001 (2005).

[21] C. N. Danson et al., Nucl. Fusion 44, S239 (2004).

[22] R. Fonseca, Lecture Notes in Computer Science (Springer, New York, 2002), Vol. 2329, pp. III-342.

[23] P. Mora, Phys. Rev. Lett. 90, 185002 (2003).

[24] S. Gitomer et al., Phys. Fluids 29, 2679 (1986). 